\title{
Improved detection of DNA Schistosoma haematobium from eggs extracted by bead beating in urine
}

\author{
Elena Pomari ${ }^{1}$ (D) Francesca Perandin ${ }^{1}$. Giulia La Marca ${ }^{1} \cdot$ Zeno Bisoffi $^{1,2}$
}

Received: 6 August 2018 / Accepted: 6 November 2018 / Published online: 12 November 2018

(C) The Author(s) 2018

\begin{abstract}
Diagnosis of Schistosoma haematobium relies primarily on microscopical analysis of urine. The method is time consuming and requires some expertise. Genus-specific real-time PCRs have been developed, but we still observed low sensitivity. In the present study, in order to achieve a more sensitive DNA detection of eggs of $S$. haematobium in urine samples, we wanted to develop a novel protocol of DNA extraction using mechanic disruption of eggs by bead beating as supplementary step. We tested Schistosoma spp. internal transcribed spacer 2 real-time PCR after both methods with and without bead beating. First, we preliminary assessed the DNA detection after bead beating using dilution of 2, 10, 50, and $90 \mathrm{eggs} / 10 \mathrm{~mL}$, and the Ct value analysis showed significant improved DNA detection per each point of egg concentration using the novel supplementary step. Twenty microscopy positive and five microscopy negative urine samples were used to validate the procedure. All urines came from imported cases and admitted at center for tropical medicine, and were examined by microscopy. PCR results after novel method with bead beating showed $100 \%$ to be positive for S. haematobium, compared with $85 \%$ positive by our standard extraction procedure. Results confirmed mechanic disruption of eggs by bead beating before DNA extraction to be highly effective method for the detection of S. haematobium DNA in urine.
\end{abstract}

Keywords Schistosoma haematobium $\cdot$ Real-time PCR $\cdot$ DNA $\cdot$ Bead beating

\section{Introduction}

Schistosomiasis, one of the most important neglected tropical diseases, is caused by parasitic trematode worms of the genus Schistosoma. Three species are of particular relevance: S. haematobium (causing urogenital schistosomiasis), S. mansoni, and S. japonicum (causing intestinal schistosomiasis) (Negussu et al. 2017). Almost 240 million people are infected worldwide and about 700 million people are at risk

Handling editor: Julia Walochnik

Electronic supplementary material The online version of this article (https://doi.org/10.1007/s00436-018-6137-7) contains supplementary material, which is available to authorized users.

Elena Pomari

elena.pomari@gmail.com

1 Centro per le Malattie Tropicali, IRCCS Ospedale Sacro Cuore Don Calabria, Via Sempreboni 5, I-37024 Negrar, VR, Italy

2 Department of Diagnostics and Public Health, University of Verona, Verona, Italy of this infection (Steinmann et al. 2006). Schistosomiasis from $S$. haematobium may remain asymptomatic for years, or just cause an often neglected, transient hematuria, before causing irreversible urogenital complications or even bladder cancer. A proper diagnosis is thus essential during the chronic phase, in order to prevent such severe problems.

Microscopic detection of Schistosoma eggs in stool and/or urine remains the gold standard for diagnosis (Sady et al. 2015). However, this method has low sensitivity (40-60\%), in relation with the intensity of the infection, the number of samples collected and the circadian and day to day variation of egg counts (Aryeetey et al. 2013; Pillay et al. 2014). The PCR technology demonstrated to be a worthy alternative to microscopy-based diagnostic methods, particularly real-time PCR, with a high sensitivity and specificity (Verweij 2014). Various Schistosoma PCRs have been described (Cnops et al. 2013; Obeng et al. 2008; Sady et al. 2015; Vinkeles Melchers et al. 2014). From 2015, we adopted a real-time PCR using a set of probe/primers specific for internal transcribed spacer 2 (ITS2) Schistosoma spp. (Obeng et al. 2008). However, we have disappointingly observed a low sensitivity of the realtime PCR that resulted positive only in about half of 
microscopic-confirmed S. haematobium infections. In order to resolve this issue and to optimize our routine molecular testing, we focused our attention on recent pieces of evidence showing improvement in DNA extraction from intestinal parasites in fecal samples using the bead beating (Kaisar et al. 2017; Liu et al. 2013; Llewellyn et al. 2016). We hypothesized that mechanic disruption of eggs by bead beating might improve DNA extraction and detection from urinary samples. The purpose of the present study was to develop a novel protocol of DNA extraction using bead beating in order to improve the DNA detection of $S$. haematobium eggs in urine.

\section{Methods}

The competent ethics committee (Comitato Etico for Clinical Research of Verona and Rovigo Provinces) approved this study (no. 25309) in April 2018. All included patients $(n=$ 25 ) signed an informed consent form for the donation of their biological samples for research purpose at our center. All patients included in this study were migrants from African endemic areas. Parasitological diagnosis was performed using standard microscopy on unpreserved urine samples collected in 120-mL container between 11:00 am and 12:00 pm when Schistosoma eggs excretion is known to be highest (Aryeetey et al. 2013; Pillay et al. 2014). A 9-mL aliquot of each sample was centrifuged for $5 \mathrm{~min}$ at $3000 \mathrm{rpm}$. Next, two aliquots $(200 \mu \mathrm{L})$ of the mixed pellet were collected; one was transferred into a 2-ml screw-capped tube without beads and one into a 2-ml screw-capped tube with ceramic beads (Roche), and frozen at $-20{ }^{\circ} \mathrm{C}$ (Kenguele et al. 2014). For microscopy, the remaining milliliter of urine was examined by the urine filtration $(25-\mathrm{mm}$ diameter and $12-\mu \mathrm{m}$ pore size filter, Whatman Nuclepore), and results were expressed as corrected number of eggs/10 mL for the volume examined. Egg counts were categorized into no infection (no eggs $/ 10 \mathrm{~mL}$ ), low

Table 1 Comparison of mean cycle threshold $(\mathrm{Ct})$ and standard deviation (SD) values between sample preparation procedures with or without bead beating

\begin{tabular}{llll}
\hline $\begin{array}{l}N \text { eggs/10 mL } \\
\text { (microscopy counts) }\end{array}$ & $N$ samples & $\begin{array}{l}\text { Ct A_PCR vs Ct B_PCR } \\
\text { mean }( \pm \text { SD) }\end{array}$ & $P$ value \\
\hline 0 & 5 & NV vs NV & NA \\
$1-2$ & 3 & NV vs $28( \pm 3.60)$ & NA \\
$1-2$ & 6 & $33( \pm 4.02)$ vs $30( \pm 3.53)$ & ns \\
$5-49$ & 8 & $31( \pm 3.51)$ vs $25( \pm 1.90)$ & 0.0007 \\
$\geq 50$ & 3 & $29( \pm 1.76)$ vs 22 $( \pm 1.15)$ & 0.0049 \\
\hline
\end{tabular}

A PCR, PCR resulted from directly frozen sample; B PCR, PCR resulted from bead beating supplemented on frozen sample; $\bar{N} V$, negative value; $N A$, not applicable. Unpaired parametric two-tailed Student's $t$ test (GraphPad Prism v7.02) was performed, and the $P$ value $\leq 0.050$ was considered significant; $n s$, not significant
$(1<50$ eggs $/ 10 \mathrm{~mL})$, and high $(\geq 50$ eggs $/ 10 \mathrm{~mL})$ infection intensities (Table 1).

For each sample, the extraction of DNA was performed by both procedures: Procedure A as conventional method without bead beating and Procedure B as new method with bead beating (more details of procedures are described in Supplementary material). In particular, for Procedure B, eggs were disrupted by a beating process for $30 \mathrm{~s}$ at $3000 \mathrm{rpm}$ using ceramic beads and a homogenization instrument (MagNA Lyser, Roche). For both procedures A and B, the DNA was extracted using MagnaPureLC.2 instrument (Roche), following the protocol DNA I Blood Cells High performance II, using the DNA isolation kit I (Roche) with a final elution volume of $100 \mu \mathrm{L}$.

Then, each DNA specimen extracted was analyzed by the Schistosoma spp. ITS2 Taqman real-time PCR (Obeng et al. 2008). The reactions, detection, and data analysis were performed with the CFX96 detection system (BioRad). The PCR reactions were considered negative/non-detected if the $\mathrm{Ct}$ value exceeded 40. Each run contained one negative (no DNA) and one S. haematobium DNA positive control. Each sample was analyzed in triplicate. As internal control for PCR inhibitors and amplification quality, the Phocine Herpes Virus type1 (PhHV-1) DNA was amplified with specific primers/probe set as multiplex PCR (Niesters 2002).

\section{Results and discussion}

We preliminary assessed the DNA detection after bead beating using dilution of $2,10,50$, and $90 \mathrm{eggs} / 10 \mathrm{~mL}$ of 3 urine samples with 131,113 , and 95 eggs/ $10 \mathrm{~mL}$ starting counts. For each sample of dilution, the extraction of DNA was performed by both procedures: Procedure A as conventional

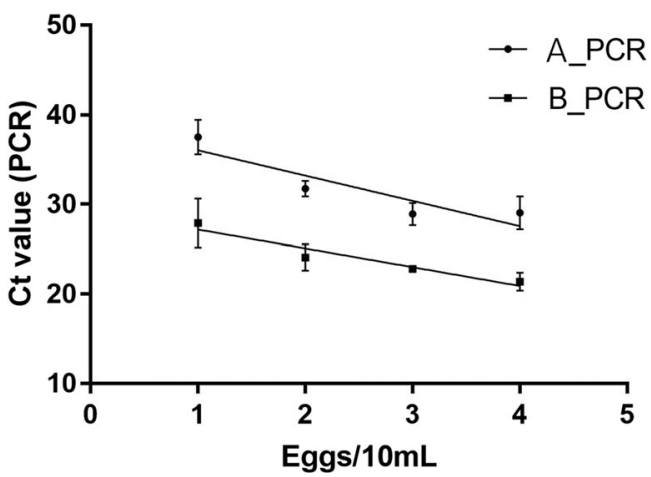

Fig. 1 Real-time PCR results and Ct values for evaluating the sensitivity with and without bead beating for DNA extraction from $S$. haematobium eggs in urine. Increasing number of eggs/10 mL was used: 2, 10, 50, and 90. Experiments were performed in triplicate and PCR results are expressed as mean of $\mathrm{Ct}$ values (GraphPad Prism v7.02). Each egg sample was processed by both procedures: A_PCR, directly frozen sample; B PCR, bead beating supplemented on frozen sample 
Fig. 2 DNA load distribution of $n=20$ S. haematobium egg samples using two different preparation procedures $\mathrm{A}$ and $\mathrm{B}$ on urine samples. PCR results following the sample preparation procedure: A PCR, directly frozen sample; B_PCR, bead beating supplemented on frozen sample

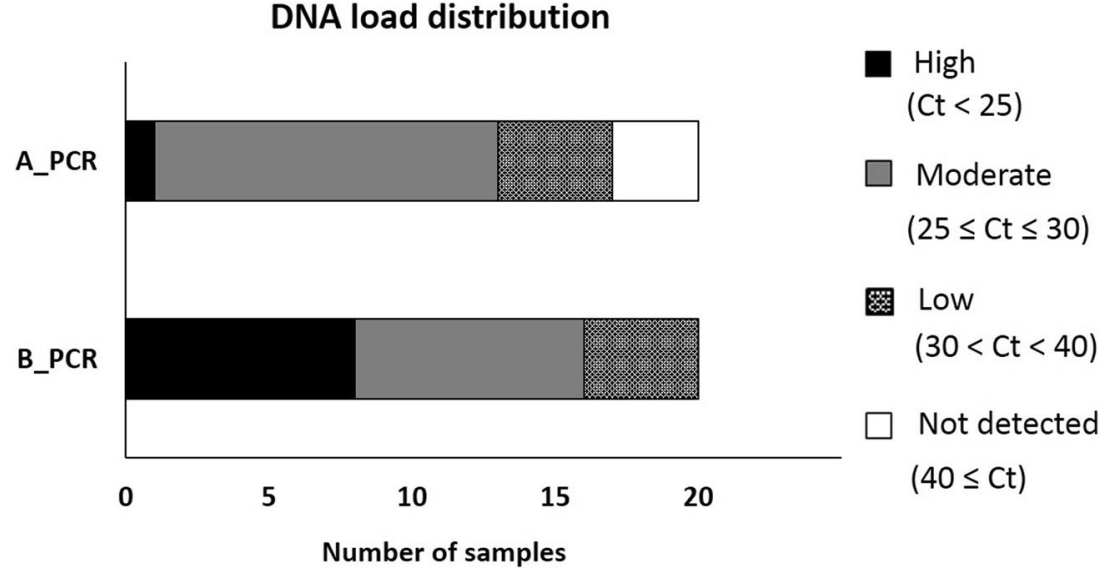

method without bead beating and Procedure B as new method with bead beating. The quality of DNA was tested by NanoVue Spectrophotometer (GE Healthcare) and good quality with A260/A280 ratio of 1.8-2.0 was obtained in all samples processed by both procedures $\mathrm{A}$ and $\mathrm{B}$.

Then, each DNA specimen extracted from this preliminary assessment was analyzed by the Schistosoma spp. ITS2 Taqman real-time PCR (Obeng et al. 2008). As internal control for PCR inhibitors and amplification quality, the PhHV-1 analysis showed the expected $\mathrm{Ct} \leq 32$ and the amplification of each sample was considered not hampered by inhibitory factors. The $\mathrm{Ct}$ values were plotted against the starting eggs equivalent per reaction by performing linear regression analysis, and $R^{2}$ of 0.92 and 0.82 was obtained from real-time PCR assay after extraction of DNA with and without bead beating, respectively (Fig. 1). Results showed the goodness of data fit of PCR assays for both procedures A and B, suggesting that the DNA detection becomes more effective independently from the PCR assay performance. Indeed, we calculated $\Delta \mathrm{Ct}$ (Ct_Procedure A-Ct_Procedure B) per each point of egg concentration and we obtained $\Delta \mathrm{Ct} 9,8,6$, and 8 respectively for $2,10,50$, and $90 \mathrm{eggs} / 10 \mathrm{~mL}$, confirming the increase of DNA detection after bead beating.

Based on these results, we extended the comparison of DNA extraction with and without bead beating on 20 undiluted microscopy positive urine samples. We used 5 urine samples with no eggs detected at microscopy as true negative. For DNA extraction and real-time PCR, we followed identical conditions of procedures $\mathrm{A}$ and $\mathrm{B}$ as defined for the preliminary data described above. Among the 20 positive samples, we observed 5\% high DNA load values $(\mathrm{Ct}<25), 60 \%$ moderate $(25 \leq \mathrm{Ct} \leq 30), 20 \%$ low $(30<\mathrm{Ct}<40)$, and $15 \%$ not detected using the procedure $\mathrm{A}$, while the procedure $\mathrm{B}$ showed $40 \%$ high, $40 \%$ moderate, and $20 \%$ low DNA load values, as shown in Fig. 2. Of note, only the procedure B provided $100 \%$ positivity. Table 1 reports the comparison between the median $\mathrm{Ct}$ values of the procedures with and without bead beating. The supplementary step of bead beating in procedure B showed a significant improved DNA detection in all samples analyzed. Moreover, the TaqMan assay resulted in specific detection of Schistosoma without any false-positive results, as shown by no DNA detection as expected on 5 negative urine samples. Similarly, negative controls (no DNA) provided no detection in each run (data not shown).

Schistosomiasis, mainly from S. mansoni and $S$. haematobium, is observed in non-endemic countries with increasing frequency. In Italy, the recent wave of asylum seekers from highly endemic areas has caused a "hidden epidemic" (Beltrame et al. 2017), with thousands of cases mostly undiagnosed, estimated to be currently living in the country. The sensitivity of direct microscopic examination as well as that of most antibody and antigen detection methods (such as CCA and CAA, mainly used for S. mansoni diagnosis) is unsatisfactory, as conventional PCR or real-time PCR, probably due to a lower average parasitic load if compared with endemic countries. The present findings suggest that a bead-beating procedure prior to DNA extraction has the potential to greatly increase $S$. haematobium DNA yield from urine. For the first assessment of this method, we used a limited sample size; a large-scale, prospective cohort study may provide more conclusive results from this method before indicating its routine use for the screening and diagnosis of imported urinary schistosomiasis. It would also be advisable that laboratories participate in an external quality assessment scheme, preferably a scheme using a proficiency panel of genuine clinical samples, to find out whether their DNA isolation procedure is sufficiently efficient.

Acknowledgments The authors would like to thank Monica Degani, Stefano Tais, Eleonora Rizzi, and Barbara Pajola for their contributions to this study.

\section{Compliance with ethical standards}

Conflict of interest The authors declare that they have no conflict of interest. 
Open Access This article is distributed under the terms of the Creative Commons Attribution 4.0 International License (http:// creativecommons.org/licenses/by/4.0/), which permits unrestricted use, distribution, and reproduction in any medium, provided you give appropriate credit to the original author(s) and the source, provide a link to the Creative Commons license, and indicate if changes were made.

\section{References}

Aryeetey YA et al (2013) Molecular diagnosis of Schistosoma infections in urine samples of school children in Ghana. The American journal of tropical medicine and hygiene 88(6):1028-1031. https://doi.org/ 10.4269/ajtmh.12-0571

Beltrame A, Buonfrate D, Gobbi F, Angheben A, Marchese V, Monteiro GB, Bisoffi Z (2017) The hidden epidemic of schistosomiasis in recent African immigrants and asylum seekers to Italy. Eur J Epidemiol 32(8):733-735. https://doi.org/10.1007/s10654-0170259-6

Cnops L, Soentjens P, Clerinx J, van Esbroeck M (2013) A Schistosoma haematobium-specific real-time PCR for diagnosis of urogenital schistosomiasis in serum samples of international travelers and migrants. PLoS Negl Trop Dis 7(8):e2413. https://doi.org/10.1371/ journal.pntd.0002413

Kaisar MMM et al (2017) Improved diagnosis of Trichuris trichiura by using a bead-beating procedure on ethanol preserved stool samples prior to DNA isolation and the performance of multiplex real-time PCR for intestinal parasites. Parasitology 144(7):965-974

Kenguele HM et al (2014) Impact of short-time urine freezing on the sensitivity of an established Schistosoma real-time PCR assay. The American journal of tropical medicine and hygiene 90(6):11531155. https://doi.org/10.4269/ajtmh.14-0005

Liu J, Gratz J, Amour C, Kibiki G, Becker S, Janaki L, Verweij JJ, Taniuchi M, Sobuz SU, Haque R, Haverstick DM, Houpt ER (2013) A laboratory-developed TaqMan Array Card for simultaneous detection of 19 enteropathogens. J Clin Microbiol 51(2): 472-480. https://doi.org/10.1128/JCM.02658-12

Llewellyn S, Inpankaew T, Nery SV, Gray DJ, Verweij JJ, Clements ACA, Gomes SJ, Traub R, McCarthy JS (2016) Application of a multiplex quantitative PCR to assess prevalence and intensity of intestinal parasite infections in a controlled clinical trial. PLoS Negl Trop Dis 10(1):e0004380. https://doi.org/10.1371/journal. pntd. 0004380

Negussu N, Mengistu B, Kebede B, Deribe K, Ejigu E, Tadesse G, Mekete K, Sileshi M (2017) Ethiopia schistosomiasis and soiltransmitted helminthes control programme: progress and prospects. Ethiop Med J 55(Suppl 1):75-80

Niesters HG (2002) Clinical virology in real time. Journal of clinical virology : the official publication of the Pan American Society for Clinical Virology 25(Suppl 3):S3-S12

Obeng BB, Aryeetey YA, de Dood CJ, Amoah AS, Larbi IA, Deelder AM, Yazdanbakhsh M, Hartgers FC, Boakye DA, Verweij JJ, van Dam GJ, van Lieshout L (2008) Application of a circulating-cathodic-antigen (CCA) strip test and real-time PCR, in comparison with microscopy, for the detection of Schistosoma haematobium in urine samples from Ghana. Ann Trop Med Parasitol 102(7):625-633

Pillay P et al (2014) Real-time polymerase chain reaction for detection of Schistosoma DNA in small-volume urine samples reflects focal distribution of urogenital Schistosomiasis in primary school girls in KwaZulu Natal, South Africa. The American journal of tropical medicine and hygiene 90(3):546-552. https://doi.org/10.4269/ ajtmh.13-0406

Sady H, al-Mekhlafi H, Ngui R, Atroosh W, al-Delaimy A, Nasr N, Dawaki S, Abdulsalam A, Ithoi I, Lim Y, Chua K, Surin J (2015) Detection of Schistosoma mansoni and Schistosoma haematobium by real-time PCR with high resolution melting analysis. Int $\mathrm{J}$ Mol Sci 16(7):16085-16103. https://doi.org/10.3390/ijms160716085

Steinmann P, Keiser J, Bos R, Tanner M, Utzinger J (2006) Schistosomiasis and water resources development: systematic review, meta-analysis, and estimates of people at risk. Lancet Infect Dis 6(7):411-425. https://doi.org/10.1016/S1473-3099(06)70521-7

Verweij JJ (2014) Application of PCR-based methods for diagnosis of intestinal parasitic infections in the clinical laboratory. Parasitology 141(14):1863-1872. https://doi.org/10.1017/S0031182014000419

Vinkeles Melchers NV et al (2014) Diagnostic performance of Schistosoma real-time PCR in urine samples from Kenyan children infected with Schistosoma haematobium: day-to-day variation and follow-up after praziquantel treatment. PLoS Negl Trop Dis 8(4): e2807. https://doi.org/10.1371/journal.pntd.0002807 